Original Research Article

\title{
Comparative study of effectiveness and safety of nonsteroidal anti- inflammatory drugs in patients of knee joint osteoarthritis of knee, tertiary care hospital, Bidar, India
}

\author{
Vivekanand, G. P. Kulkarni*
}

Department of Pharmacology, Bidar Institute of Medical Sciences, Bidar, Karnataka, India

Received: 29 November 2018 Accepted: 28 December 2018

*Correspondence to:

Dr. G. P. Kulkarni,

Email: dr.vivek499@gmail.com

Copyright: (C) the author(s), publisher and licensee Medip Academy. This is an openaccess article distributed under the terms of the Creative Commons Attribution NonCommercial License, which permits unrestricted noncommercial use, distribution, and reproduction in any medium, provided the original work is properly cited.

\begin{abstract}
Background: Osteoarthritis (OA) is a joint failure and OA is the most frequent chronic joint disease causing pain and disability. Where all the structures of joints have undergone pathological changes and they are hyaline articular cartilage loss which may be focal or non-uniform, initially it will be focal then spread all over non-uniformly. Non-Steroidal Anti Inflammatory Drugs (NSAID) are the mainstay of medical management of OA. Increased in reports suggests that GIT adverse effect with old NSAID's and cardiovascular effects with selective cyclooxygenase-2 (COX2) inhibitors had precipitated to chase for better NSAID's with minimal adverse effects. The current study compares the clinical effectiveness and safety of newer NSAID's, etoricoxib, lornoxicam, to diclofenac which has been standard therapy in patients of OA of the knee joint.

Methods: The current study is randomized, prospective, open-label, parallel group study conducted in 120 patients with OA of the knee joint diagnosed using American College of Rheumatology criteria. After getting the informed consent, they were randomized in three groups of 40 patients each who received tablet etoricoxib 120mg BID, tablet Lornoxicam 16mg BID, tablet diclofenac 50mg TID respectively. The duration of the study is 12 weeks. Data are calculated, tabulated and analyzed using analysis of variance (ANOVA) test, and level of significance was determined by its $\mathrm{P}$ value.

Results: After 12 weeks of treatment, the severity of pain and functional indices using visual analog scale and Western Ontario and McMaster Universities Osteoarthritis score were significantly better $(\mathrm{P}<0.05)$ in etoricoxib group as compared to lornoxicam or diclofenac group along with a lesser rate of adverse effects.

Conclusions: It is concluded that etoricoxib is more effective and tolerated NSAID than lornoxicam and diclofenac in the treatment of knee joint OA.
\end{abstract}

Keywords: Diclofenac, Etoricoxib, Lornoxicam, VAS, WOMAC Score

\section{INTRODUCTION}

The main pathogenic mechanism of Osteoarthritis $(\mathrm{OA})$ is suggested as wearing and destruction of cartilage tissue due to ageing. But it is described as an active and dynamic process with destruction and repair triggered by biochemical and mechanical factors, nowadays. ${ }^{1}$ Osteoarthritis (OA) is also known as a chronic progressive disease of the weight-bearing joints characterized by degeneration of articular cartilage, subchondral bony plate sclerosis, osteophyte, and cyst formation. These pathological changes will result clinically in pain, stiffness, crepitus, swelling, limited movement leading to significant disability in weight bearing joints, loss of productivity, and impaired quality of life..$^{2,3}$

The mainstay treatment of $\mathrm{OA}$ requires a combination of pharmacological and non-pharmacological therapy. ${ }^{4}$ The present pharmacological methods are mostly acting as symptomatically relief but do not stop the progression of OA. Surgical procedures, such as arthroplasty and osteotomy, provide encouraging results when appropriate patients are selected, and good surgical techniques are applied..$^{5}$ 
Treatment for $\mathrm{OA}$ targets at reducing pain, maintaining mobility, and minimization of disability. Non-Steroidal Anti Inflammatory Drugs are the mainstay on medical management of OA. ${ }^{6}$ Increased in reports about GIT adverse effects with traditional NSAID's and cardiovascular effects with selective cyclooxygenase-2 (COX-2) inhibitors had precipitated to chase for better tolerated NSAID's with minimal adverse effects.

Etoricoxib is having 30 times more selective for COX-2 inhibitor. It is used for chronic inflammatory conditions such as osteoarthritis, chronic low backache, gouty arthritis, ankylosing spondylitis, and also in acute inflammatory conditions. Increase in use of etoricoxib by prescription as well as self-administered routes has led to increase in reports of side effects and adverse reactions including dermatologic reactions in $0.1 \%-0.3 \%$ of cases. ${ }^{7}$

\section{METHODS}

The study conducted was a comparative, randomized, prospective, open label, parallel group study in patients of knee joint OA diagnosed according to the American College of Rheumatology (ACR) criteria. The study protocol was approved by the institutional thesis committee and the institutional ethics committee before the study was initiated. Informed consent was obtained from all patients included in the study after being informed about the nature of the study. This study was conducted in accordance with the Principles of Good Clinical Practice and Declaration of Helsinki.

\section{The investigational drugs for this study were:}

- Tablet etoricoxib 120mg B.I.D.

- Tablet lornoxicam 16mg B.I.D.

- Tablet diclofenac sodium 50mg T.I.D.

\section{Inclusion criteria}

Patients who were found fit in baseline examination were included in the present study as per the criteria given below.

- $\quad$ Patients with symptoms of OA for $>3$ months, whose diagnosis of OA the knee had been diagnosed by the clinical examination and $\mathrm{X}$-rays of the knee joint according to the ACR criteria

- Patients of OA of knee joint who were already on different NSAIDs or other analgesic medications were included after a washout period of 4 weeks.

\section{Exclusion criteria}

- Age $<50$ years or $>75$ years of either gender.

- Patients with hepatic or renal impairment or concomitant active gastro duodenal ulcers, within last one month before receiving the drug under study.

- Patients with known hypersensitivity or contraindication for any NSAIDs.
- Patients on therapy with warfarin or heparin or high dose aspirin (>1000mg/day).

- Women of either pregnant or lactating or on oral contraceptive pills.

- Patients with known cardiovascular disorder or uncontrolled hypertension or ischemic heart disease or patients who had undergone coronary artery bypass graft or angioplasty.

- Patients with history of transient ischemic attacks, myocardial infarction, stroke, within previous 3years.

- Patients diagnosed with other arthritis, gout, or sustained acute trauma to knee, hip, or spine.

- History of arthroscopy of affected knee within 6 months before to include in the study.

- History of acute meniscal injury or ligamentous injury to study joint within previous 2years.

- $\quad$ Patients with severe knee deformity

Consenting patients with fulfilling the eligibility criteria, previously diagnosed with OA and previously taking any NSAID's were given a washout period of 4 weeks. After including in to the study, patients were assessed and randomly allocated to receive per oral (P.O) either tablet etoricoxib 120mg B.I.D in group A, or tablet lornoxicam 20mg B.I.D in group B, and tablet Diclofenac sodium 50 $\mathrm{mg}$ T.I.D in group $\mathrm{C}$.

Drug treatment, all history about past, personal, and family history, any concomitant illness, vital signs were recorded. Patients were re-evaluated at 4, 8, and 12 weeks after starting treatment in accordance with the Visual Analog Scale (VAS), Western Ontario and McMaster Universities Osteoarthritis index (WOMAC), X-rays, and physician's and patient's global assessment. The total period of treatment was 12 weeks.

The primary endpoint was clinically improvement in pain, disability, and range of movement. The secondary endpoint was patients and physician's assessment, VAS and WOMAC composite scores. Adverse effects and concomitant use of drugs if any were recorded throughout the study. For every adverse effect, the severity, outcome, and causal relationship to treatment were recorded.

\section{Statistical analysis}

All the data generated from this study were tabulated and expressed as and then analyzed statistically using analysis of variance (ANOVA) tests. The statistical analysis of the data was performed using the SPSS statistical package. The level of significance was calculated by its $P$ value. $P$ $<0.05$ was considered to be statistically significant.

\section{RESULTS}

Out of total 150 patients screened, 130 are found eligible to be included in the study and are randomized to either of Group A, B, or C receiving P.O. etoricoxib 120mg B.I.D, Lornoxicam $20 \mathrm{mg}$ B.I.D, or diclofenac $50 \mathrm{mg}$ T.I.D, 
respectively. A total of 40 patients in each group completed the treatment period of 12 weeks to form the per protocol population.

This study male predominance is seen with male to female proportionate of $2.6(\mathrm{M}: \mathrm{F}=2.6)$, with $87(72.5 \%)$ are male patients and remaining are female. The following figure explain about gender distribution (Figure 1).

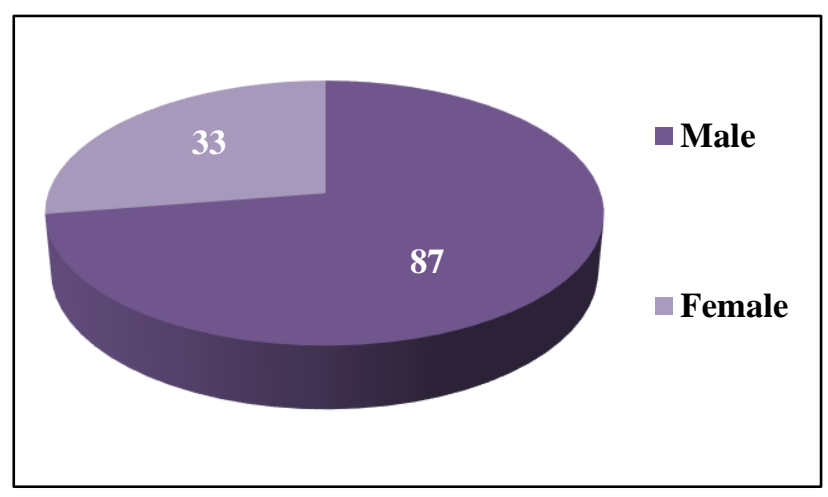

Figure 1: Gender distribution.

Table 1: Reduction in percentage VAS scores in all the patients among all three groups.

\begin{tabular}{|lllll|}
\hline & Before & $\mathbf{4}^{\text {th }}$ & $\mathbf{8}^{\text {th }}$ & $\mathbf{1 2}^{\text {th }}$ \\
\hline \multirow{2}{*}{ Etoricoxib } & \multirow{2}{*}{7.98} & $\begin{array}{l}7.05 \\
(11.89 \%)\end{array}$ & $\begin{array}{l}5.02 \\
(37.30 \%)\end{array}$ & $\begin{array}{l}3.54 \\
(55.72 \%)\end{array}$ \\
\hline \multirow{2}{*}{ Lornoxicam } & \multirow{2}{*}{7.98} & $\begin{array}{l}6.50 \\
(18.76 \%)\end{array}$ & $\begin{array}{l}5.48 \\
(31.52 \%)\end{array}$ & $\begin{array}{l}4.51 \\
(43.58 \%)\end{array}$ \\
\hline \multirow{2}{*}{ Diclofenac } & \multirow{2}{*}{7.98} & $\begin{array}{l}6.54 \\
(18.23 \%)\end{array}$ & $\begin{array}{l}6.54 \\
(18.23 \%)\end{array}$ & $\begin{array}{l}5.48 \\
(31.52 \%)\end{array}$ \\
\hline
\end{tabular}

The reduction in percentage VAS scores of all the group before treatment is almost equal without any significant difference and that is 7.98 , in etoricoxib group is reduced by $7.05(11.89 \%)$ at week $4,5.02(37.30 \%)$ at week 8 , and $3.54(55.72 \%)$ at week 12. In Lornoxicam group, the reduction in mean VAS scores is by $6.50(18.76 \%)$ at week $4,5.48(31.52 \%)$ at week 8 , and $4.51(43.58 \%)$ at week 12 . In Diclofenac group, the reduction in mean VAS scores is by $6.54(18.23 \%)$ at week $4,6.54(18.23 \%)$ at week 8 , and $5.48(31.52 \%)$ at week 12 . At the end of study period of 12 weeks, there is a high significant $(\mathrm{P}<0.001)$ decrease in VAS scores in all the three groups (Table 1).
Table 2: Reduction in percentage WOMAC score in all the patients among all three groups.

\begin{tabular}{|c|c|c|c|c|}
\hline & Before & $4^{\text {th }}$ & $8^{\text {th }}$ & $12^{\text {th }}$ \\
\hline Etoricoxib & 44 & $\begin{array}{l}38 \\
(13.64 \%)\end{array}$ & $\begin{array}{l}34 \\
(22.73 \%)\end{array}$ & $\begin{array}{l}25 \\
(43.18 \%)\end{array}$ \\
\hline Lornoxicam & 44 & $\begin{array}{l}40 \\
(9.09 \%)\end{array}$ & $\begin{array}{l}38 \\
(13.64 \%)\end{array}$ & $\begin{array}{l}34 \\
(22.73 \%)\end{array}$ \\
\hline Diclofenac & 44 & $\begin{array}{l}41 \\
(6.82 \%)\end{array}$ & $\begin{array}{l}36 \\
(18.18 \%)\end{array}$ & $\begin{array}{l}35 \\
(20.45 \%)\end{array}$ \\
\hline
\end{tabular}

The reduction in percentage WOMAC score (total 44), in etoricoxib group is by $38(13.64 \%)$ at week $4,34(22.73 \%)$ at week 8 , and $25(43.18 \%)$ at week 12. In lornoxicam group, the reduction in mean WOMAC score (total 44) is by $40(9.09 \%)$ at week $4,38(13.64 \%)$ at week 8 , and 34 $(22.73 \%)$ at week 12 . In diclofenac group, the reduction in mean WOMAC score (total 44$)$ is by $41(6.82 \%)$ at week 4, $36(18.18 \%)$ at week 8, and $35(20.45 \%)$ at week 12 (Table 2).

In patient's global assessment at 12 weeks, 5 (12.5\%) patient in etoricoxib group assessed their condition as "very good" whereas $3(7.5 \%)$ patients in lornoxicam group and $1(2.5 \%)$ patients in diclofenac group respectively. $22(55 \%)$ patients in etoricoxib group assessed their condition as "good" whereas 18 (45\%) patients in lornoxicam group and $12(30 \%)$ patients in diclofenac group respectively. $10(25 \%)$ patient in etoricoxib group assessed their condition as "fair" whereas $12(30 \%)$ patients in lornoxicam group and $19(47.5 \%)$ patients in diclofenac group respectively. $3(7.5 \%)$ patient in etoricoxib group assessed their condition as "poor" whereas $7(17.5 \%)$ patients in lornoxicam group and 5 $(12.5 \%)$ patients in diclofenac group respectively. Only 3 $(7.5 \%)$ in diclofenac group were assessed as "very poor". (P<0.05) (Table 3).

In physician's global assessment at 12 weeks, physician assessed $24(60 \%)$ patients in etoricoxib group as "very good" as compared to $14(35 \%)$ patients in Lornoxicam group and $10(25 \%)$ patients in Diclofenac group. Also, 16 $(40 \%)$ patients in etoricoxib group, $26(65 \%)$ patients in Lornoxicam group, and $22(55 \%)$ patients in Diclofenac group were assessed as "good" with statistically significant difference between the groups $(\mathrm{P}<0.05) .8(20 \%)$ patients in diclofenac group were assessed as "fair"(Table 4).

Table 3: Improvement in patient's global assessment in all the patients in all three groups.

\begin{tabular}{|lllll|}
\hline Patient's global assessment & Etoricoxib group & Lornoxicam group & Diclofenac group & Total \\
\hline Very Good & $5(12.5 \%)$ & $3(7.5 \%)$ & $1(2.5 \%)$ & 9 \\
\hline Good & $22(55 \%)$ & $18(45 \%)$ & $12(30 \%)$ & 52 \\
\hline Fair & $10(25 \%)$ & $12(30 \%)$ & $19(47.5 \%)$ & 41 \\
\hline Poor & $3(7.5 \%)$ & $7(17.5 \%)$ & $5(12.5 \%)$ & 15 \\
\hline Very poor & 0 & 0 & $3(7.5 \%)$ & 3 \\
\hline & 40 & 40 & 40 & 120 \\
\hline
\end{tabular}


Table 4: Improvement in physician global assessment in all the patients in all three groups.

\begin{tabular}{|lllll|}
\hline Patient's global assessment & Etoricoxib group & Lornoxicam group & Diclofenac group & Total \\
\hline Very good & $24(60 \%)$ & $14(35 \%)$ & $10(25 \%)$ & 48 \\
\hline Good & $16(40 \%)$ & $26(65 \%)$ & $22(55 \%)$ & 64 \\
\hline Fair & 0 & 0 & $8(20 \%)$ & 8 \\
\hline Poor & 0 & 0 & 0 & 0 \\
\hline Very poor & 0 & 0 & 0 & 0 \\
\hline & 40 & 40 & 40 & 120 \\
\hline
\end{tabular}

Table 5: Adverse Drug Reaction occurred in all the patients among all three groups.

\begin{tabular}{|llll|}
\hline & $\begin{array}{l}\text { Etoricoxib } \\
\text { group }\end{array}$ & $\begin{array}{l}\text { Lornoxicam } \\
\text { group }\end{array}$ & $\begin{array}{l}\text { Diclofenac } \\
\text { group }\end{array}$ \\
\hline $\begin{array}{l}\text { Nausea and } \\
\text { vomiting }\end{array}$ & $10(25 \%)$ & $13(32.5 \%)$ & $20(50 \%)$ \\
\hline $\begin{array}{l}\text { Abdomen } \\
\text { pain }\end{array}$ & $7(17.5 \%)$ & $6(15 \%)$ & $8(20 \%)$ \\
\hline Dyspepsia & $3(7.5 \%)$ & $3(7.5 \%)$ & $6(15 \%)$ \\
\hline $\begin{array}{l}\text { Aphthous } \\
\text { ulcer }\end{array}$ & $2(5 \%)$ & 0 & 0 \\
\hline Headache & 0 & $2(5 \%)$ & $2(5 \%)$ \\
\hline
\end{tabular}

All the study drugs are better-tolerated with no serious adverse events requiring hospitalization. Adverse events usually of gastrointestinal tract (GIT) are often a limiting factor for NSAID use, comprising of mild events such as dyspepsia and nausea.

Nausea and vomiting are observed in $20(50 \%)$ patients in diclofenac group whereas $13(32.5 \%)$ patients in lornoxicam group and $10(25 \%)$ patients in etoricoxib group respectively. Abdominal pain is observed in $8(20 \%)$ patients in diclofenac group whereas $6(15 \%)$ patients in lornoxicam group and $7(17.5 \%)$ patients in etoricoxib group respectively. Dyspepsia are observed in $6(15 \%)$ patients in diclofenac group whereas $3(7.5 \%)$ patients in each lornoxicam and etoricoxib group. $2(5 \%)$ patients in etoricoxib group presented with Aphthous ulcer. 2 (5\%) patients presents with headache in each Lornoxicam and etoricoxib group (Table 5).

\section{DISCUSSION}

Forty patients from each group completed the treatment period of 12 weeks to form the per-protocol population in Group A, B, and C receiving P.O. Etoricoxib 120mg B.I.D, Lornoxicam20mg B.I.D, or Diclofenac 50mg T.I.D, respectively.

The progress in symptoms and functional status of each patient with these drugs was assessed at 4,8 , and 12 weeks of treatment for different parameters such as respective mean Visual Analog Scale and WOMAC scores and the percentage change in mean scores. Baseline score of Visual Analog Scale and WOMAC was related in the three groups with no significant difference $(\mathrm{P}>0.05)$. Over the 12-week study period, all three drugs provided significantly $(\mathrm{P}<0.05)$ sustained comfortably in symptoms of $\mathrm{OA}$ as compared with baseline. The number of patients showing progress in different parameters was significant in all the three groups $(\mathrm{P}<0.05)$.

After 12 weeks of treatment, the reduction in the percentage VAS score of etoricoxib group (55.72\%) is significantly more than lornoxicam (43.58\%) and diclofenac group $(31.49 \%)$. At the end of the study, there is a significant $(\mathrm{P}<0.05)$ reduction in overall pain individually in all the groups. On comparing the three groups together, the reduction in mean VAS score is highly significant $(\mathrm{P}<0.001)$ between diclofenac and lornoxicam group, however significant $(\mathrm{P}<0.05)$ between diclofenac and etoricoxib group.

The results in this study are relatively comparable to those obtained in randomized, controlled clinical trial comparing efficacy, safety, and cost-effectiveness of lornoxicam with Diclofenac sodium in patients of OA of knee joint which reported that $80.89 \%$ reduction in pain in lornoxicam group as compared to $45.45 \%$ reduction in diclofenac sodium group at 3 months $(\mathrm{P}<0.001)$. Furthermore, the mean pain score of lornoxicam group is less than the diclofenac group after 2 and 3 months of treatment. ${ }^{8}$ Similar results are also obtained in another 12-week study in 135 patients of arthritis where those treated with lornoxicam $8 \mathrm{mg}$ T.I.D. and $8 \mathrm{mg}$ B.I.D. conveyed same clinical efficacy $(\mathrm{P}<0.05)$ as diclofenac $50 \mathrm{mg}$ T.I.D and the clinical effects remained stable, with more pain reduction of up to $23 \%$ in the course of a subsequent 40-week follow-up treatment in lornoxicam group. ${ }^{9}$

In present study, percentage WOMAC Score (total) after 12 weeks of treatment in etoricoxib group $(43.18 \%)$ is significantly less than lornoxicam (22.73\%)) and Diclofenac group (20.45\%).

The reduction in WOMAC scores (total) of current study are in accordance with the results of another study conducted to compare clinical effectiveness and tolerability of oral lornoxicam $8 \mathrm{mg}$ B.I.D. and diclofenac 50mg T.I.D. in adult Indian patients with OA knee or hip joint. ${ }^{10}$ On analysing the data at 12 weeks, significantly difference $(\mathrm{P}<0.05)$ in the scores of VAS and WOMAC 
was observed in both etoricoxib group and lornoxicam group as compared to Diclofenac group with maximum reduction VAS and WOMAC scores in etoricoxib group. Similar results were obtained in another study conducted to compare effectiveness and tolerability of oral lornoxicam $8 \mathrm{mg}$ B.I.D. and Diclofenac $50 \mathrm{mg}$ T.I.D. in Indian patients of OA of the knee or hip joint. ${ }^{10}$ Patient's global assessment was seen similarly $(\mathrm{P}>0.05)$ in the three treatment groups at baseline evaluation with progressively over the 12 weeks study period.

At the end of 12 weeks, assessments were in favour of etoricoxib group with $22(55 \%)$ patients assessing their condition as "good" as compared to 18 (45\%) in Lornoxicam group and $12(30 \%)$ patients in Diclofenac group. However, 10 (25\%) patients in etoricoxib group, 12 (30\%) patients in Lornoxicam group, and 19 (47.5\%) patients in Diclofenac group assessed their condition as "fair" and $3(7.5 \%)$ patients in etoricoxib group, 7 (17.5\%) patients in lornoxicam group, and $5(12.5 \%)$ patients in Diclofenac group assessed their condition as "poor" and 3 (7.5\%) patient in Diclofenac group assessed their condition as "very poor". Pair-wise comparisons revealed significantly more improvement from baseline scores $(\mathrm{P}$ $<0.05)$ for etoricoxib and lornoxicam group than in Diclofenac group with significant intergroup difference ( $\mathrm{P}$ $<0.05)$.

At baseline evaluation, the physician's global assessment was similar $(\mathrm{P}>0.05)$ in the three treatment groups which improved progressively over 12 weeks. Most of the patients had "poor" or "fair" condition at baseline with no significant intergroup difference $(\mathrm{P}>0.05)$. At 12 weeks, physician's assessment was in favour of etoricoxib group with $24(60 \%)$ assessed as "very good" as compared to 14 $(35 \%)$ patients in lornoxicam group and $10(25 \%)$ patients in diclofenac group. A total of $16(40 \%)$ patients in etoricoxib group, $26(65 \%)$ patients in lornoxicam group, and $22(55 \%)$ patients in diclofenac group were assessed as "good" with statistically significant difference between the groups $(\mathrm{P}<0.05)$.

Significant progress from baseline was reported in all patients of the three groups $(\mathrm{P}<0.05)$. At the end of study period, physician's and patient's assessment were in favour of etoricoxib-treated group as compared to lornoxicam and diclofenac-treated group with significant difference between the three groups $(\mathrm{P}<0.05)$. The results of our study are comparable with a previous 12 -week multicentre, randomized, parallel group study done to compare the efficacy and tolerability of lornoxicam and diclofenac in treatment of patients with OA of hip and/or knee with significantly intergroup equivalence $(\mathrm{P}<0.033)$ in terms of progress in disease activity (about $46 \%$ ), pain severity (42\%-48\%), patient's and physician's global assessments. ${ }^{9}$ Similar results were seen in other double-blind studies where lornoxicam was compared to other NSAIDs such as diclofenac, piroxicam, and indomethacin in patients with knee OA in terms of physician's and patient's overall assessments. ${ }^{11,12}$
In present study, all the study drugs were well-tolerated without any severe adverse events requiring hospitalization. Adverse events of the GIT are often a limiting factor for NSAID use such as dyspepsia and nausea which are eventually associated with reduced tolerability and noncompliance to the treatment protocol. ${ }^{13}$ Better tolerability is probably to result in a high level of compliance which was $90 \%$ in our study as confirmed by counting tablets and empty blister packs. The most common adverse effects reported include mild-to-moderate degree GIT disturbances usually manifested as pain abdomen, dyspepsia, flatulence, nausea with a mild headache, or insomnia which was significantly $(\mathrm{P}<0.05)$ less in etoricoxib and lornoxicam group than in diclofenac group.

None of the patients reported with cardiovascular adverse drug reactions (e.g., hypertension or oedema) or any hepatic function abnormality.

In present study, etoricoxib approved to be an effective alternative in the treatment of $\mathrm{OA}$, as illustrated by significantly better progress in pain and functional indices of VAS and WOMAC, limited use of rescue medication, and satisfactory patient's and physician's global assessment.

\section{ACKNOWLEDGEMENTS}

Authors grateful to the Department of Orthopaedic for their constant encouragement, valuable insight and facilities at all stages of this work.

Funding: No funding sources

Conflict of interest: None declared

Ethical approval: The study was approved by the Institutional Ethics Committee

\section{REFERENCES}

1. Sarıdoğan ME. Cerrahpaşa Medical Faculty, Continuing Medical Education Activities Rheumatic diseases Symposium Series. 2003;34:11-8.

2. Creamer P, Hochberg MC. Osteoarthritis. Lancet. 1997;350:5038.

3. Brandt KD. Osteoarthritis. Clin Geriatr Med. 1988;4:279-93.

4. Baker JF, Walsh P, Mulhall KJ. Statins: a potential role in the management of osteoarthritis? Joint Bone Spine. 2011 Jan 1;78(1):31-4.

5. Verbruggen G. Chondroprotective drugs in degenerative joint diseases. Rheumatology (Oxford). 2006 Feb;45(2):129-38.

6. Fawaz-Estrup F. The osteoarthritis initiative: an overview. Med Health R I. 2004 Jun;87(6):169-71.

7. Settipane GA. Aspirin and allergic diseases: a review. Am J Med. 1983 Jun 14;74(6):102-9.

8. Vadgama VK, Gharia R, Mehta K, Tripathi CB. A randomized controlled clinical trial comparing efficacy safety and cost effectiveness of lornoxicam 
with diclofenac sodium in patients of osteoarthritis knee. Internet J Med Update. 2011;6(2):25-9.

9. Kidd B, Frenzel W. A multicenter, randomized, double blind study comparing lornoxicam with diclofenac in osteoarthritis. J Rheumatol. 1996 Sep;23(9):1605-11.

10. Goregaonkar A, Mathiazhagan KJ, Shah RR, Kapoor PS, Taneja P, Sharma A, et al. Comparative assessment of the effectiveness and tolerability of lornoxicam $8 \mathrm{mg}$ BID and diclofenac $50 \mathrm{mg}$ TID in adult indian patients with osteoarthritis of the hip or knee: a 4-week, double-blind, randomized, comparative, multicenter study. Curr Therap Res. 2009 Feb 1;70(1):56-68.

11. Karbowski A. Double-blind, parallel comparison of etodolac and indomethacin in patients with osteoarthritis of the knee. Curr Med Res Opin. 1991 Jan 1;12(5):309-17.

12. Gomes Freitas G. A double-blind comparison of etodolac and piroxicam in the treatment of osteoarthritis. Curr Med Res Opin. 1990 Jan 1;12(4):255-62.

13. Singh G, Triadafilopoulos G. Epidemiology of NSAID induced gastrointestinal complications. J Rheumatol Suppl. 1999 Apr;56:18-24.

Cite this article as: Vivekanand, Kulkarni GP. Comparative study of effectiveness and safety of nonsteroidal anti-inflammatory drugs in patients of knee joint osteoarthritis of knee, tertiary care hospital, Bidar, India. Int J Basic Clin Pharmacol 2019;8:181-6. 